\title{
EDITORIAL
}

\section{A new generation of comorbidity research in the era of neuroscience and Research Domain Criteria}

\author{
THEODORE P. BEAUCHAINE ${ }^{a}$ AND DANTE CICCHETTI ${ }^{b, c}$ \\ ${ }^{a}$ The Ohio State University; ${ }^{b}$ University of Minnesota Institute of Child Development; and ${ }^{c}$ University of Rochester Mt. Hope \\ Family Center
}

When the revised third edition of the Diagnostic and Statistical Manual of Mental Disorders (DSM-III-R; American Psychiatric Association [APA], 1987) was published 30 years ago, few could have anticipated the fundamental changes to psychopathology research and practice that would ensue. The DSM-III-R was released so soon after the DSM-III (APA, 1980), and contained so few changes to diagnostic criteria, that it could not be considered a fourth edition. Nevertheless, one change, elimination of exclusion criteria, altered the way that child and adult psychopathologists conceptualized, diagnosed, and even treated psychiatric disorders (see Beauchaine \& Klein, in press). Exclusion criteria were used to implement diagnostic hierarchies, which aid in differential diagnosis. Because those who are afflicted with psychopathology often present with a variety of symptoms, most of which are nonspecific to any single disorder, the task of diagnosis can be formidable, and in some cases somewhat arbitrary. Diagnostic hierarchies assign certain symptoms priority over others, which simplifies diagnostic decisions. In the DSM-III, organic mental disorders were at the top of the diagnostic hierarchy, followed by schizophrenia, major mood disorders, and neurotic/personality disorders. According to this hierarchy, if an organic brain syndrome (central nervous system disease, brain trauma, or substanceinduced brain damage) was identified, diagnoses of schizophrenia, mood disorders, and neurotic/personality disorders were precluded. In the absence of organic factors, schizophrenia received priority in diagnosis, even if symptoms of mood and/or neurotic/personality disorders were present. In the absence of both organic factors and schizophrenia, mood disorder symptoms took precedence. Neurotic/personality disorder

Address correspondence and reprint requests to: Theodore P. Beauchaine, Department of Psychology, The Ohio State University, 1835 Neil Avenue, Columbus, OH 43210; E-mail: beauchaine.1@ osu.edu; or Dante Cicchetti, Institute of Child Development, University of Minnesota, 51 East River Road, Minneapolis, MN 55455; E-mail: cicchett@umn.edu. diagnoses were considered only in the absence of organic brain syndromes, schizophrenia, and mood disorders.

Research conducted in the early 1980s demonstrated that for some disorders, excluding symptoms at lower levels of the diagnostic hierarchy omitted important information, with potential implications for understanding etiology and devising effective treatments (e.g., Leckman, Weissman, Merikangas, Pauls, \& Prusoff, 1983). Although details of such studies are beyond the scope of this editorial, they prompted the APA to abandon diagnostic hierarchies in subsequent versions of the DSM, except when used to rule out organic (e.g., general medical or substance-induced) causes of mental disorder.

It is perhaps not surprising that diagnostic hierarchies curtailed rates of comorbidity. Individuals could not be diagnosed with schizophrenia and depression, or with a mood disorder and a personality disorder. This situation changed markedly following publication of the DSM-III-R. In the subsequent decade or so, foundational articles on potential sources and subtypes of comorbidity emerged (e.g., Angold, Costello, \& Erkanli, 1999; Klein \& Riso, 1993), comorbidity became a major focus of study, and the National Comorbidity Survey (NCS), a congressionally mandated evaluation of DSM-III-R psychiatric disorders and their co-occurrence, was launched (Kessler et al., 1994). Understanding comorbidity has been at the top of the research agenda of mental health professionals, including developmental psychopathologists, ever since (see, e.g., Beauchaine \& McNulty, 2013).

The NCS, which was conducted between 1990 and 1992, indicated higher prevalence rates of psychopathology than were expected at the time. Almost $50 \%$ of respondents reported a lifetime DSM-III-R psychiatric disorder (Kessler et al., 1994), and among this group, half experienced at least one additional disorder and a third experienced three or more disorders. These findings initiated an upsurge in comorbidity research that continues to this day. More recent efforts to document patterns of comorbidity include the NCS-2, which included reinterviews of NCS respondents to evaluate the 
course of mental illness (see Kessler \& Walters, 2002); the NCS Replication Survey, which evaluated patterns of comorbidity among an entirely new nationally representative sample using DSM-IV (APA, 1994) diagnostic criteria (see Kessler et al., 2005); and the NCS Adolescent Supplement, which was conducted with a large, nationally representative sample of 13- to 18-year-olds (see Merikangas et al., 2010).

These large-scale studies, and others conducted with smaller samples of children, adolescents, and adults, indicate high rates of homotypic comorbidity for both internalizing and externalizing disorders, and high rates of heterotypic comorbidity between internalizing and externalizing disorders (see Beauchaine \& McNulty, 2013). Furthermore, longitudinal studies indicate substantial heterotypic continuity of psychopathology across development. For example, a sizable proportion of impulsive preschoolers with attention-deficit/ hyperactivity disorder progress to increasingly severe forms of externalizing conduct across development and experience occupational and relational impairment into adulthood (Beauchaine, Hinshaw, \& Pang, 2010). Similarly, early life anxiety portends considerable vulnerability to later depressive disorders and continued impairment across the life span (Emmelkamp \& Wittchen, 2009). In both cases, progression to more severe forms of psychopathology is mediated by complex ontogenic processes through which individual level vulnerabilities interact with environmental adversities to canalize developmental pathways to disorder (Beauchaine \& McNulty, 2013; Cicchetti, Rogosch, \& Toth, 1997; Cicchetti \& Toth, 1998).

Most early comorbidity research was descriptive. Respondents or their caretakers were interviewed carefully, and rates of diagnostic co-occurrence were tabulated. This research was important, because painstaking description is a necessary antecedent to understanding any natural phenomenon (Beauchaine \& Klein, in press). However, description alone is rarely if ever sufficient for full understanding of such phenomena. Rather, description poses questions that can only be resolved by identifying causal mechanisms (Beauchaine, Gatzke-Kopp, \& Mead, 2007). Early studies of comorbidity raised a number of important questions about the nature of psychopathology, and about developmental, mechanistic processes through which psychopathology emerges. Among the most fundamental of these questions is whether distinctions among psychiatric disorders, as currently defined, are valid. Exceedingly high rates of homotypic comorbidity, and well-characterized heterotypic progressions along the internalizing and externalizing spectra, suggest that at least some assumedly separate DSM disorders are alternative developmental manifestations of common etiologies. Externalizing spectrum disorders, for example, including attentiondeficit/hyperactivity disorder, oppositional defiant disorder, conduct disorder, antisocial personality disorder, and substance use disorder, share genetic, temperamental, subcortical, and cortical vulnerabilities (see Beauchaine \& Hinshaw, 2016). However, as behavioral repertoires change across development, and as environmental adversities accumulate and interact with biological vulnerabilities, the expression externalizing behavior changes.

This general pattern, whereby biological vulnerabilities interact with environmental adversities to eventuate in expanding and changing symptom expression across development, is observed in almost all multifactorially inherited disorders, whether mental or physical. For example, abnormal glucose tolerance, which interacts with environmental risk to eventuate in Type II diabetes, is a highly heritable multifactorial trait (Poulsen, Ohm Kyvik, Vaag, \& Beck-Nielsen, 1999). The developmental progression of Type II diabetes follows a predictable course that begins with insulin resistance, followed by weight gain, elevated triglycerides, high blood pressure, nonalcoholic fatty liver disease, inflammation, ischemic heart disease, kidney failure, retinal damage, and peripheral neuropathy. Thus, observable symptoms of Type II diabetes increase in complexity and expand in scope across the life span. It is important to note, however, that abnormal glucose tolerance may never develop into Type II diabetes and its adverse tertiary outcomes in protective environments characterized by healthy diet, controlled weight, low stress, and exercise. Similarly, early life impulsivity may never develop into more severe psychopathology in protective environments characterized by secure attachment relationships, effective parenting, neighborhood cohesion, and positive peer groups. Yet unlike the case of externalizing spectrum disorders, the pathophysiology of Type II diabetes is well understood. As a result, even though an individual in an advanced stage of illness expresses a far more progressive and variegated set of symptoms than an individual who is early in the course of illness, we do not attribute accruing symptoms to independent health conditions, as we often do with heterotypically continuous psychopathological disorders for which etiology and pathophysiology are at best only partly understood. From this perspective, advanced symptoms observed following lifelong progression along the externalizing spectrum are tertiary expressions of vulnerability that occur only if development of impulsivity is not arrested by altering environmental risk mediators (see Beauchaine \& McNulty, 2013). This developmental conceptualization of externalizing conduct, in which progressively more severe symptoms are viewed as outcomes of complex ontogenic processes rather than independent disorders, has profound implications for future models of comorbidity.

In the era of early comorbidity research, etiological and pathophysiological models of psychopathology were far less advanced than they are today. Nevertheless, studies of comorbidity demonstrated clearly that most externalizing spectrum disorders, most internalizing spectrum disorders, and many externalizing and internalizing disorders are not independent of one another. This conclusion is consistent with extensive factor analytic and behavioral genetics findings, including research from prominent developmental psychopathologists (e.g., Achenbach \& Edelbrock, 1983), that consistently indicate a hierarchical latent structure of psychopathology in which (a) higher order internalizing and exter- 
nalizing factors account for most of the covariation among lower order syndromes (i.e., disorders), and (b) higher order internalizing and externalizing factors are themselves correlated (see Zisner \& Beauchaine, 2016).

Taken together, patterns of comorbidity and the well-replicated factor structure of psychopathology suggest common pathophysiologies among behavioral syndromes that are often considered to be distinct, and imply that a limited number of traits, which are far fewer in quantity than disorders in our diagnostic system, interact to confer vulnerability to psychopathology. This supposition undergirds the Research Domain Criteria (RDoC) initiative, which specifies five major domains of behavior (negative valence, positive valence, cognitive, social, and arousal/regulatory), which are assumed to interact with one another to affect temperament and personality, and at their extremes, psychopathology (see Beauchaine \& Klein, in press; Kozak \& Cuthbert, 2016). Consistent with work that has long been advanced by this journal (e.g., Beauchaine, 2001; Burnette \& Cicchetti, 2012; Cicchetti \& Natsuaki, 2014; Cicchetti \& Thomas, 2008), and with longstanding biobehavioral motivational systems perspectives with roots in the psychophysiology literature (see Beauchaine \& Thayer, 2015), an explicit goal of RDoC is to identify biobehavioral vulnerabilities that cut across currently defined disorders. Although RDoC is not developmentally focused, this objective has clear implications for improved understanding of comorbidities and continuities in psychopathology. As noted above, the transdiagnostic vulnerability approach has already led to improved understanding of the development of externalizing conduct (see Beauchaine \& Hinshaw, 2016).

An obvious question that emerges from this discussion concerns the neurobiological bases of these vulnerability traits. When comorbidity research first exploded three decades ago, neuroimaging research, at least as applied to human behavior, was in its infancy. Thus, even though prominent neurobiological models of trait vulnerabilities to psychopathology existed (e.g., Fowles, 1988; Gray, 1982; Porges, 1995), they were difficult to confirm or falsify. Today, the neurobiological bases of subcortical emotion generation systems and cortical behavior/ emotion regulation systems, both of which are implicated in numerous forms of psychopathology, have been studied extensively (see Beauchaine, 2015). However, research has only begun to emerge that evaluates interactive effects of these brain systems/networks on comorbidity (e.g., Sauder, Beauchaine, Gatzke-Kopp, Shannon, \& Aylward, 2012). To advance our

\section{References}

Achenbach, T. M., \& Edelbrock, C. (1983). Manual for the Child Behavior Checklist and revised child behavior profile. Burlington, VT: University of Vermont, Department of Psychiatry.

American Psychiatric Association. (1980). Diagnostic and statistical manual of mental disorders (3rd ed.). Washington, DC: Author.

American Psychiatric Association. (1987). Diagnostic and statistical manual of mental disorders (3rd ed., revised). Washington, DC: Author.

American Psychiatric Association. (1994). Diagnostic and statistical manual of mental disorders (4th ed.). Washington, DC: Author. understanding of comorbidity, it will be important for future research to move beyond main effects models of brain-behavior relations to examine interactive associations among multiple brain networks and co-occurring conditions.

A multiple neural systems approach to comorbidity research will also address questions concerning why certain conditions, including externalizing disorders, depression, and self-inflicted injury, share core neural vulnerabilities (Sauder, Derbidge, \& Beauchaine, 2016; Zisner \& Beauchaine, 2016), yet are expressed quite differently in most contexts. Multiple levels of analysis research has already identified mechanisms of neural plasticity, allostasis, epigenesis, and central nervous system insult that mediate environmentally induced alterations in function among neural systems implicated in impulsivity, social affiliation, and emotion regulation (e.g., Beauchaine, Neuhaus, Zalewski, Crowell, \& Potapova, 2011; Cicchetti, 2006, 2015; Cicchetti, Ackerman, \& Izard, 1995; Cicchetti \& Dawson, 2002; Crews, He, \& Hodge, 2007). Through these mechanisms, environmental adversity often hastens progression of psychopathology among vulnerable individuals, promoting heterotypic continuity (Beauchaine $\&$ McNulty, 2013). Thus, understanding comorbidity and continuity is not simply a matter of identifying genetic and neural vulnerabilities. It is therefore important for future research to evaluate complex Biology $\times$ Environment interactions in the development of psychopathology, its comorbidities, and its continuities. Such ontogenic processes are not captured well by RDoC and will need to be identified and characterized by developmental psychopathologists.

Descriptive research on comorbidity has provided us with important next questions about psychopathology that can only be addressed through a new generation of multiple levels of analysis research that identifies etiological and pathophysiological mechanisms, and specifies how environmental adversities interact with such mechanisms to promote concurrent comorbidity and heterotypic continuity. Continued conceptualization of DSM-defined disorders as independent diagnostic entities will almost certainly impede these objectives (Beauchaine \& McNulty, 2013). We hope that this Special Issue, in which authors were encouraged to move beyond simple cross tabulation of symptoms to identify and/or speculate about causal mechanisms, helps to launch the next generation of comorbidity research in which satisfactory mechanisms for already well-characterized patterns of symptom overlap are identified. After all, identifying causal mechanisms is the ultimate goal of science (Popper, 1985).

Angold, A., Costello, E. J., \& Erkanli, A. (1999). Comorbidity. Journal of Child Psychology and Psychiatry, 40, 57-87.

Beauchaine, T. P. (2001). Vagal tone, development, and Gray's motivational theory: Toward an integrated model of autonomic nervous system functioning in psychopathology. Development and Psychopathology, 13, 183-214.

Beauchaine, T. P. (2015). Future directions in emotion dysregulation and youth psychopathology. Journal of Clinical Child and Adolescent Psychology, 44, 875-896. 
Beauchaine, T. P., Gatzke-Kopp, L., \& Mead, H. K. (2007). Polyvagal theory and developmental psychopathology: Emotion dysregulation and conduct problems from preschool to adolescence. Biological Psychology, 74, 174-184.

Beauchaine, T. P., \& Hinshaw, S. P. (Eds.). (2016). The Oxford handbook of externalizing spectrum disorders. New York: Oxford University Press.

Beauchaine, T. P., Hinshaw, S. P., \& Pang, K. L. (2010). Comorbidity of attention-deficit/hyperactivity disorder and early-onset conduct disorder: Biological, environmental, and developmental mechanisms. Clinical Psychology: Science and Practice, 17, 327-336.

Beauchaine, T. P., \& Klein, D. N. (in press). Classifying psychopathology: The DSM, empirically-based taxonomies, and the research domain criteria. In T. P. Beauchaine \& S. P. Hinshaw (Eds.), Child and adolescent psychopathology (3rd ed.). Hoboken, NJ: Wiley.

Beauchaine, T. P., \& McNulty, T. (2013). Comorbidities and continuities as ontogenic processes: Toward a developmental spectrum model of externalizing behavior. Development and Psychopathology, 25, 1505-1528.

Beauchaine, T. P., Neuhaus, E., Zalewski, M., Crowell, S. E., \& Potapova, N. (2011). Effects of allostatic load on neural systems subserving motivation, mood regulation, and social affiliation. Development and Psychopathology, 23, 975-999.

Beauchaine, T. P., \& Thayer, J. F. (2015). Heart rate variability as a transdiagnostic biomarker of psychopathology. International Journal of Psychophysiology, 98, 338-350.

Burnette, M. L., \& Cicchetti, D. (2012). Multilevel approaches toward understanding antisocial behavior: Current research and future directions. Development and Psychopathology, 24, 703-704.

Cicchetti, D. (2006). Development and psychopathology. In D. Cicchetti \& D. J. Cohen (Eds.), Developmental psychopathology (Vol. 1, 2nd ed., pp. 1-23). Hoboken, NJ: Wiley.

Cicchetti, D. (2015). Neural plasticity, sensitive periods, and psychopathology. Development and Psychopathology, 27, 319-320.

Cicchetti, D., Ackerman, B., \& Izard, C. (1995). Emotions and emotion regulation in developmental psychopathology. Development and Psychopathology, 7, 1-10.

Cicchetti, D., \& Dawson, G. (2002). Multiple levels of analysis. Development and Psychopathology, 14, 417-420.

Cicchetti, D., \& Natsuaki, M. N. (2014). Multilevel developmental perspectives toward understanding internalizing psychopathology: Current research and future directions. Development and Psychopathology, 26, 1189-1190.

Cicchetti, D., Rogosch, F. A., \& Toth, S. L. (1997). Ontogenesis, depressotypic organization, and the depressive spectrum. In S. S. Luthar, J. Burack, D. Cicchetti, \& J. Weisz (Eds.), Developmental psychopathology: Perspectives on adjustment, risk, and disorder (pp. 273-313). New York: Cambridge University Press.

Cicchetti, D., \& Thomas, K. M. (2008). Imaging brain systems in normality and psychopathology. Development and Psychopathology, 20, 1023-1027.

Cicchetti, D., \& Toth, S. L. (1998). The development of depression in children and adolescents. American Psychologist, 53, 221-241.

Crews, F., He, J., \& Hodge, C. (2007). Adolescent cortical development: A critical period of vulnerability for addiction. Pharmacology Biochemistry and Behavior, 86, 189-199.

Emmelkamp, P. M. G., \& Wittchen, H. U. (2009). Specific phobias. In G. Andrews, D. S. Charney, Paul. J. Sirovatka, \& D. A. Regier (Eds.), Stress-in- duced and fear circuitry disorders: Refining the research agenda for DSM$V$ (pp. 77-101). Arlington, VA: American Psychiatric Association.

Fowles, D. C. (1988). Psychophysiology and psychopathology: A motivational approach. Psychophysiology, 25, 373-391.

Gray, J. A. (1982). The neuropsychology of anxiety: An enquiry into the function of the septo-hippocampal system. New York: Oxford University Press.

Kessler, R. C., Berglund, P., Demler, O., Jin, R., Merikangas, K. R., \& Walters, E. E. (2005). Lifetime prevalence and age-of-onset distributions of DSM-IV disorders in the National Comorbidity Survey replication. Archives of General Psychiatry, 62, 593-602.

Kessler, R. C., McGonagle, K. A., Zhao, S., Nelson, C. B., Hughes, M., Eshleman, S., et al. (1994). Lifetime and 12-month prevalence of DSM-III-R psychiatric disorders in the United States. Archives of General Psychiatry, 51, 8-19.

Kessler, R. C., \& Walters, E. E. (2002). The National Comorbidity Survey. In M. T. Tsaung \& M. Tohen (Eds.), Textbook in psychiatric epidemiology (2nd ed., pp. 343-362). New York: Wiley.

Klein, D. N., \& Riso, L. P. (1993). Psychiatric disorders: Problems of boundaries and comorbidity. In C. G. Costello (Ed.), Basic issues in psychopathology (pp. 19-66). New York: Guilford Press.

Kozak, M. J., \& Cuthbert, B. N. (2016). The NIMH Research Domain Criteria initiative: Background, issues, and pragmatics. Psychophysiology, 53, 286-297.

Leckman, J. F., Weissman, M. M., Merikangas, K. R., Pauls, D. L., \& Prusoff, B. A. (1983). Panic disorder and major depression: Increased risk of depression, alcoholism, panic, and phobic disorders in families of depressed probands with panic disorder. Archives of General Psychiatry, $40,1055-1060$.

Merikangas, K. R., He, J., Burstein, M., Swanson, S. A., Avenevoli, S., Cui, L., et al. (2010). Lifetime prevalence of mental disorders in U.S. adolescents: Results from the National Comorbidity Survey replication-Adolescent supplement (NCS-A). Journal of the American Academy of Child \& Adolescent Psychiatry, 49, 980-989.

Popper, K. R. (1985). The aim of science. In D. Miller (Ed.), Popper selections (pp. 162-170). Princeton, NJ: Princeton University Press. (Original work published 1957)

Porges, S. W. (1995). Orienting in a defensive world: Mammalian modifications of our evolutionary heritage: A polyvagal theory. Psychophysiology, 32, 301-318.

Poulsen, P., Ohm Kyvik, K., Vaag, A., \& Beck-Nielsen, H. (1999). Heritability of Type II (non-insulin-dependent) diabetes mellitus and abnormal glucose tolerance-A population-based twin study. Diabetologia, 42, 139-145.

Sauder, C., Beauchaine, T. P., Gatzke-Kopp, L. M., Shannon, K. E., \& Aylward, E. (2012). Neuroanatomical correlates of heterotypic comorbidity in externalizing male adolescents. Journal of Clinical Child and Adolescent Psychology, 41, 346-352.

Sauder, C. L., Derbidge, C. M., \& Beauchaine, T. P. (2016). Neural responses to monetary incentives among self-injuring adolescent girls. Development and Psychopathology, 28, 277-291.

Zisner, A., \& Beauchaine, T. P. (2016). Neural substrates of trait impulsivity, anhedonia, and irritability: Mechanisms of heterotypic comorbidity between externalizing disorders and unipolar depression. Development and Psychopathology, 28, 1179-1210. 